\title{
Who takes care of the kids at when? Variation of sex-role patterns across different parental care forms in passerine birds
}

\author{
Daiping Wang ${ }^{1 *}$ and Xiang-Yi Li Richter ${ }^{2 *}$
}

${ }^{1}$ Key Laboratory of Animal Ecology and Conservation Biology, Institute of Zoology, Chinese Academy of Sciences, 1 Beichen West Road, Chaoyang, 100101 Beijing, China

${ }^{2}$ Institute of Biology, University of Neuchâtel, Rue Emile-Argand 11, CH-2000 Neuchâtel, Switzerland

*correspondence author, email: wangdaiping@ioz.ac.cn; email: li@evolbio.mpg.de

Keywords: parental care, nest building, nest incubation, offspring provisioning, sex roles, sexual selection, certainty of paternity, passerine birds

Figures \& Tables: 2 figures, 1 table

Supplementary materials: 2 tables

\section{Abstract (< 200 words):}

Parental care in birds usually consists of many elaborate forms, including nest building, incubation, provisioning the offspring and protecting them. Given the various life history differences between sexes, parents may have different opportunity costs of providing care in a specific form. But we still do not know whether males and females generally differ in their involvement in different care forms across stages of a breeding cycle, such as nest building, incubation and chick provisioning. Here, we performed a survey of parental care regarding which sex provides care in 882 species of passerine birds and found significant differences in the frequency distributions of sex-role patterns (i.e. female-only care, biparental care, and male-only care) across three distinct forms of parental care. This result showed clearly that parental care should not be treated as a unitary trait, but a composite of integrated features with diverse functions. Using a set of linear mixed-effect models we tested the effects of sexual selection, certainty of paternity, predation risk, and offspring's life history traits in driving the variation in sex roles. In general, we found species with female-only care tended to be under strong sexual selection on males, and uncertainty of paternity could reduce male care. 


\section{Introduction}

Birds often provide extensive parental care that enhances the survival and future reproductive fitness of their offspring. Avian parental care comprises diverse forms, including nest building, incubation, provisioning the offspring and defending them against predators [1-3]. Despite the benefits that parental care brings, it costs energy, time, the opportunity for extra-pair mating and/or starting a new clutch, and may increase the predation risk for the parents. Consequently, there are conflicts between parents and offspring, and between male and female parents. In species of cooperative breeding, the conflicts also involve helpers of different degrees of relatedness with the breeders and the dependent offspring. These intricate relationships have inspired theoretical studies about the optimal parental care strategies. Early models characterised parental care as an all-or-none choice between deserting and caring [4-7], while later models generally treated parental investment as a continuous trait. The optimal levels of parental efforts have been studied as functions of various factors, including brood quality [8,9], certainty of paternity [10-12], operational sex ratio and sexual selection [13,14], and sex-specific life history characters such as adult mortality [15] and the ability to care [16]. Special attention has been paid to how the male and female parent negotiate to determine which sex should provide care, and in the case of biparental care, the amount of effort each of them contributes [17-20]. Most theoretical work, however, treat parental care as a unitary trait rather than a composite of several functionally integrated characteristics. A few rare exceptions have considered task specialisation between parents, such as feeding the young and defending them from predators [21,22], but these models do not make predictions on how parents contribute in different tasks over time across a breeding cycle.

Do sex-specific parental strategies differ across distinct care forms? In other words, if one sex has participated in nest building, should it also incubate the eggs laid in that nest and/or feed the chicks after they hatch? Empirical studies provided some tentative hints that sex-specific opportunity costs of providing care can differ between different stages. In black coucals (Centropus grillii), a species of uniparental care by males only, incubating males were $17 \%$ less likely to sire extra-pair offspring than males that were not currently parenting, while males feeding nestlings were $48 \%$ less likely to sire extra-pair offspring than nonparenting males [23]. The varying degrees of disadvantage experienced by parenting males in siring extrapair offspring showed that the opportunity costs of parenting can differ between different forms of care at different stages for males. In the cooperatively breeding chestnut-crowned babblers (Pomatostomus ruficeps), as brood aged, breeding females contributed less food than male breeders and helpers, and were the only carer to load-lighten by reducing their provisioning rates in the presence of additional carers [24]. The adjustment of provisioning rates by breeder females suggested the conservation of resources for future reproduction has been an opportunity cost of caring for the current brood, which increased in later stages of a reproductive cycle for females [24]. Therefore, we hypothesized that the involvement of males and females in different care forms may differ across different stages of parental care.

If our expectation was correct, the next step would be to uncover possible driving forces of the variation of sex roles across different care forms. In particular, we considered factors including sexual selection, certainty 
of parentage, predation risk, and offspring's life history traits such as clutch size and the duration of nestling development. Sexual selection was an important factor to consider because it was predicted to produce female-biased care in theory models $[13,14]$, and was shown to be associated with evolutionary transitions between major patterns of parental care $[25,26]$. Sexual selection was also found to correlate negatively with the extent of parental cooperation [27]. Certainty of parentage was also an interesting factor to consider because theory and intuition generally suggested that males should invest more in the care of their genetic offspring especially when parental care is costly [10-12], but empirical support has been mixed, with frequent exceptions where males do not seem to react to paternity loss by reducing care or biasing their paternal efforts towards own genetic offspring [28-31]. We were also interested in testing the effect of predation risk because female passerine birds usually have more cryptic plumages than males [32], and therefore they might be more effective in providing care especially under high nest predation risk. The life history traits of offspring are of interest to study because they reflect broods' reproductive value and needs. Because parents' caring efforts are linked to the trade-off between their current and future reproductive fitness, they are expected to invest more in broods of higher reproductive value [8,9,33-35]. And since larger broods and offspring with longer developmental time generally need more care, we are interested in testing whether higher needs of parental care were achieved by the participation of more carers (i.e. biparental care relative to uniparental care, with helpers relative to without helpers).

To find out whether sex roles in parental care differ between distinct care forms across different stages of reproduction, we performed a survey of the participation of males and females in three care forms (i.e. nest building, incubation, and offspring provisioning) across 882 species of passerine birds. As expected, we found marked differences in the frequency distributions of parental care patterns (i.e. female-only care, biparental care, and male-only care) across three care forms. To further investigate possible causes of the above finding, we built five linear mixed-effect models to test the roles of sexual selection, certainty of paternity, nest daily predation risk, clutch size, and nestling developmental time in driving the variation of sex roles in parental care. In general, we found species with female-only care are under strong sexual selection, and uncertain of parentage could reduce male care.

\section{Materials and methods}

\section{Sex roles classification}

We surveyed all passerine species in the Birds of the World database [36] for which sex provides parental care in each of the three forms - nest building, incubation, and offspring provisioning - across a reproductive cycle. The three forms were chosen because of the affluence of data and the relatively low phylogenetic correlations between them [3]. We took notes of the parental care features for each species from the breeding section of the species account, and then classified them into four categories for each form of care: (1) 'Male care', where only paternal care was present; (2) 'Female care', where only maternal care was present; (3) 'Biparental care', where both parents provide care, and (4) 'Cooperation', where helpers of cooperatively breeding species also participate in caring of offspring (typically offspring provisioning). 
Since we are interested in the general patterns across different passerine species at an evolutionary scale, the within-species variations of sex roles were ignored. Therefore, cases where a form of care was provided usually by females alone but males were occasionally observed to participate were classified as 'Female care', and vice versa. In rare cases (34 species in nest building, 42 species in incubation, and 17 species in offspring provisioning), the parental care information was recorded with uncertain words, such as "reportedly" or "probably" in one or more care forms (e.g. White-throated Bulbul: nest reportedly built by both sexes; ... incubation possibly by both sexes, period 13 days; chicks fed by both parents). All statistical models were run by first including and then excluding those uncertain data.

In some species of cooperative breeding, the sex role categorization in each care form was straightforward (e.g. White Helmet-shrike: Cooperative breeder, all group-members assisting in all aspects of nesting duties. Breeding pair chooses nest-site and does most of the construction work, but assisted by other group members, ...; incubation by all group-members, ...; chicks brooded and fed by all of the group). In the others, the contribution of helpers to each care form may not be clearly specified. Given that cooperative breeding with helpers usually implies helpers' participation in chick provisioning [37], we classified those species' offspring provisioning as 'Cooperation', and classified the other two care forms according to additional details in the description regarding sex roles. For example, according to the description "Drakensberg Rockjumper: breeds as monogamous pair and co-operative, with helpers. Nest built by both sexes, ...; incubation by both sexes; no other information.", we classified this species' nest building and incubation as 'Biparental care', and offspring provisioning as 'Cooperation'.

Following the above procedures, we collected 882 species with 'full data' (i.e. information about sex roles in all three care forms). We then matched the scientific names used in the data source [36] with the species names from a phylogenetic information source (BirdTree.org) [38] for further statistical analyses. Finally, we included in the statistical models 868 species of passerine birds where we have complete data on the phylogenetic information and contributor(s) of parental care in nest building, incubation, and offspring provisioning.

\section{Explanatory variables in statistical models}

(a) Body size, which is the first principle component ( $\mathrm{PC} 1)$ of body mass and wing length ( $\mathrm{N}=749$ species), and (b) sexual selection, which is the PC1 of mating system and sexual dimorphism ( $\mathrm{N}=749$ species), were obtained following Dale et al. (2015) [32]. In short, the mating system was scored on a four-point scale, with ' 0 ' representing strict social monogamy (e.g. zebra finch Taeniopygia guttata), ' 1 ' representing monogamy with infrequent instances of polygyny observed ( $<5 \%$ of males, e.g. lazuli bunting Passerina amoena), ' 2 ' representing mostly social monogamy with regular occurrences of facultative social polygyny ( 5 to $20 \%$ of males, e.g. American redstart Setophaga ruticilla), and ' 3 ' representing obligate resource defense polygyny ( $>20 \%$ of males, e.g. lance-tailed manakin Chiroxiphia lanceolata). Sexual dimorphism was quantified by the difference of plumage colors between males and females [32]. (c) EPP was the proportion of extra-pair 
offspring ( $N=112$ species) and (d) EPBr was the proportion of broods with extra-pair offspring ( $N=111$ species). Data on EPP and EPBr was obtained from the study of Brouwer \& Griffith (2019) [39]. (e) Daily predation rate of nest ( $\log 10$ transformed, $N=225$ species) was obtained from Matysioková \& Remeš (2018) [40]. (f) Clutch size ( $\log 10$ transformed, $N=733$ species) and (g) length of the nestling developmental period (in days, $\log 10$ transformed, $\mathrm{N}=591$ species) were collated from Cooney et al. (2020) [41]. (h) Research effort ( $\mathrm{N}=840$ species), quantified as the number of independent entries per species in the Zoological Record database [42], was incorporated to account for data quality.

\section{Statistical analyses}

All analyses were carried out within R statistical environment [43]. Mainly, we used linear mixed-effect models from the package 'Ime4' [44] and phylogenetic regression models from the package 'phylolm' $[45,46]$ to investigate the variation of sex roles across three forms of parental care (nest building, incubation, and offspring provisioning), and tested the roles of the assumed driving forces of the variation. Given the difference of premises regarding different hypotheses and the number of species available for relevant explanatory variables, we coded the response variables (i.e. the contributor(s) of parental care in each form) in two different ways, depending on the corresponding explanatory variables in a series of mixed-effects models.

The first way of recoding the contributor(s) of parental care focuses on the which sex provides the care. We recoded 'Female care', 'Biparental care' and 'Male care' as ' -1 ', ' 0 ', and ' +1 ', respectively. Species in the 'Cooperation' category were also coded as ' 0 ', because breeders and helpers of both sexes contributed to care. Using this way of recoding, we built four models to test whether sexual selection, extra-pair paternity, and nest predation were the main driving factors determining which sex provide care in each form. The second way of recoding the contributor(s) of parental care focuses on the number of individuals that provide care to a brood in each of the three forms. In this way, we recoded 'Female care' and 'Male care' as ' 1 ', 'Biparental care' as '2', and 'Cooperation' as ' 3 ', because there were only one carer (either the male or the female) in the first category, two carers (both the male and female parent) in the second category, and at least three carers (both the male and female breeder and at least one helper) in the third category. The second way of recoding allowed us to build an additional model to test the association between offspring's life history traits (reflecting offspring's reproductive value and brood needs) and the number of carers in each care form. Detailed information about the five models were listed below.

Model 1: The model was built to quantify the association between sex roles in each of the three forms of parental care and sexual selection. In this model, sex roles of parental care (using the first way of recoding) was added as the response variable. We included 'form of care' (three levels: nest building, incubation, and offspring provisioning), 'sexual selection', 'body size', and 'research effort' as fixed effects. The 'family' and 'genus' of the species was included as a random effect to account for phylogenetic uncertainty. To fully control for phylogenetic uncertainty, we additionally used a phylogenetically controlled regression method 
as implemented in the function 'phylolm' from the $\mathrm{R}$ package phylolm [45,46]. In this model, the phylogenetic tree of species was included as a random effect, and we ran the model using 100 different phylogenetic trees from Jetz et al. (2012) [38]. Results are therefore based on mean estimates for predictor slopes and model-averaged standard errors. However, because our dataset included multiple traits for each species (i.e. sex roles in nest building, nest incubation and offspring provisioning), directly adding a phylogenetic tree into the model as a random effect seems unattainable, because the three different traits were considered as "repeated values" in the model. To solve this issue, we randomly selected one representative observation (out of three) for each species and ran the model with the culled data, which contained only one observation per species. Note that in this way, the full control for phylogeny was achieved at the cost of losing two thirds of the observations.

Model 2 and Model 3: The two models were built to assess the association between sex roles in each of the three forms of parental care and the degrees of uncertainty in paternity. Sex roles of parental care (using the first way of recoding) was added as the response variable. We included 'form of care', 'body size', 'research effort', and either 'EPP' (in Model 2) or 'EPBr' (in Model 3) as fixed effects. Regarding random effect(s), due to the limited number of species with data on extra-pair paternity (see the section of 'explanatory variables' above), the model simultaneously contained two radom effects ('family' and 'genus') caused a singular fit issue. This indicated an overfitting of the model, meaning that the random effects structure was too complex to be parameterized by the limited data. Therefore, we only included one random effect (either 'family' or 'genus') that explained more variation of the response variable. Additionally, we built two phylogenetically controlled regression models using the same fixed effects but treated the phylogenetic tree as random effect using the phylolm package as explained in Model 1.

Model 4: The model was built to test the association between sex roles in each of the three forms of parental care and daily nest predation rates. Sex roles of parental care (using the first way of recoding) was added as the response variable. We included 'daily nest predation', 'form of care', 'body size', and 'research effort' as fixed effects, and 'family' and 'genus' of the species as a random effect. Additionally, we built a complementary phylogenetically controlled regression model with the same fixed effects while using the phylogenetic tree as random effect.

Model 5: The model was built to test the association between the number of carers in different care forms and offspring's life history traits. In this model, sex roles of parental care (using the second way of recoding) was added as the response variable. We included 'form of care', 'length of nestling developmental period', 'clutch size', and 'research effort' as fixed effects. Like in the other models, 'family' and 'genus' of the species was treated as a random effect to account for phylogenetic uncertainty. Additionally, like in Models 1 to 4 , we built a complementary phylogenetically controlled regression model using the phylogenetic tree as random effect. 


\section{Results}

\section{Large variation in sex roles across different forms of parental care}

226 We found substantial variation regarding which sex provides care across three different care forms in passerine birds (incubation $v s$ nest building: $t=-12.2, p<0.0001$; offspring provisioning $v s$ nest building: $t=19.3, p<0.0001$; Figure 1; Table 1: Model 1). In general, 'Female care' and 'Biparental care' were the predominant categories across three care forms, while 'Male care' only presented in a small proportion $(2.4 \%$, $\mathrm{N}=21$ ) of species in a single form of care (nest building). Specifically, during nest building, except for a small group of species with exclusive male investment, female and biparental care occurred almost equally frequently ( $N=401$ species and $N=460$ species for female and biparental care, respectively; Figure 1). During incubation, 'Female care' was the most prevailing form ( $\mathrm{N}=585$ species; Figure 1), about twice the frequency of 'Biparental care' ( $N=297$ species; Figure 1). During offspring provisioning, 'Biparental care' was the foremost category ( $\mathrm{N}=810$ species; Figure 1), with more than ten times the frequency of 'Female care' $(\mathrm{N}=72$ species; Figure 1).

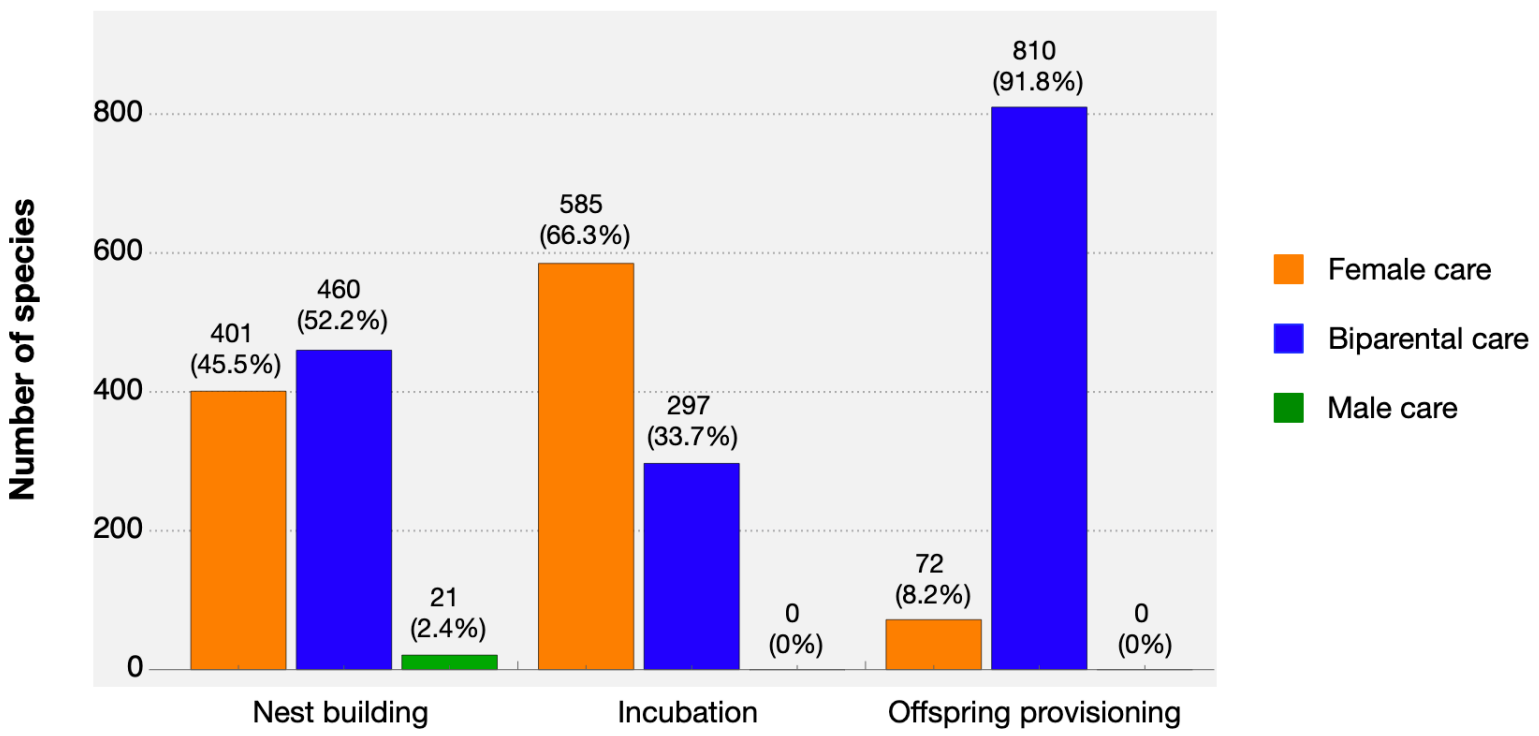

Forms of parental care

Figure 1. Variation of sex roles in different forms of parental care. The results were based on 882 species with full data on sex roles ('Female care', 'Biparental care', and 'Male care') across three forms of parental care: nest building, incubation, and offspring provisioning. Bars of different colors represent different sex role categories. Note that a few species of cooperative breeding were grouped into the 'Biparental care' category ( $\mathrm{N}=34$ species in nest building, $\mathrm{N}=21$ in incubation, and $\mathrm{N}=105$ in offspring provisioning). effect, the size of the variance components is shown. The estimate with its standard error (SE), $t$-value, and corresponding $p$ value is shown for each fixed effect. 


\begin{tabular}{|c|c|c|c|c|}
\hline \multirow[t]{2}{*}{ Model 1} & \multirow{2}{*}{\multicolumn{2}{|c|}{$\begin{array}{l}\text { Estimate } \\
(\beta \pm \mathrm{SE})\end{array}$}} & \multirow[b]{2}{*}{$\mathrm{t}$} & \multirow[b]{2}{*}{$\mathrm{P}$} \\
\hline & & & & \\
\hline \multicolumn{5}{|c|}{ Random effects: } \\
\hline & Family $(\mathrm{n}=78)$ & 0.013 & & \\
\hline & Genus $(n=300)$ & 0.04 & & \\
\hline & Residual & 0.135 & & \\
\hline \multicolumn{5}{|c|}{ Fixed effects: } \\
\hline & Intercept & $-0.412 \pm 0.030$ & -13.7 & - \\
\hline & Nest incubation & $-0.235 \pm 0.019$ & -12.2 & $<0.001$ \\
\hline & Offspring provisioning & $0.370 \pm 0.019$ & 19.3 & $<0.001$ \\
\hline & Sexual selection & $-0.066 \pm 0.001$ & -5.9 & $<0.001$ \\
\hline & Body size & $-0.054 \pm 0.016$ & -3.5 & 0.001 \\
\hline & Research effort & $0.00004 \pm 0.00003$ & 1.1 & 0.29 \\
\hline
\end{tabular}

Model 2 Random effects:

$\begin{array}{ll}\text { Family }(\mathrm{n}=42) & 0.046 \\ \text { Residual } & 0.143\end{array}$

Fixed effects:

Intercept

Nest incubation

Offspring provisioning

EPP

Research effort

Model 3 Random effects:

Family $(n=42)$

Residual

Fixed effects:

Intercept

Nest incubation

Offspring provisioning

$\mathrm{EPBr}$

Research effort

$$
\begin{aligned}
&-0.409 \pm 0.060 \\
&-0.214 \pm 0.051 \\
& 0.491 \pm 0.051 \\
& 0.004 \pm 0.002 \\
& 0.000001 \pm 0.00005 \\
& 0.046 \\
& 0.145
\end{aligned}
$$$$
-0.214 \pm 0.051
$$$$
0.491 \pm 0.051
$$$$
0.004 \pm 0.002
$$$$
-0.000001 \pm 0.00005
$$

$-6.8$

$-4.2$

9.7

$-2.3$

$-0.1$
$<0.001$

0.001

0.02

0.89

Model 4

Random effects:

Family $(\mathrm{n}=55)$

Genus ( $\mathrm{n}=132)$

Residual

$$
\begin{array}{r}
-0.397 \pm 0.063 \\
-0.225 \pm 0.051 \\
0.478 \pm 0.051 \\
-0.002 \pm 0.001 \\
0.000 \pm 0.000
\end{array}
$$

Fixed effects:

Intercept
Nest incubation
Offspring provisioning
Body size

$-11.1$

15.9 $<0.001$

$-0.8$ 
Model 5

$\begin{array}{ll}\text { Random effects: } & \\ \text { Family }(\mathrm{n}=72) & 0.069 \\ \text { Genus }(\mathrm{n}=229) & 0.044 \\ \text { Residual } & 0.165\end{array}$

Fixed effects:

Intercept

Nest incubation

$1.516 \pm 0.438$

3.5

Offspring provisioning

$-0.192 \pm 0.027$

$-7.2$

$<0.001$

Body size

$0.563 \pm 0.027$

21.2

$<0.001$

Nestling developmental

$-0.020 \pm 0.027$

$-0.8$

0.45 time

$0.035 \pm 0.279$

0.1

0.9

Clutch size

$0.06 \pm 0.128$

0.64

Research effort

$-0.00002 \pm 0.00004$

0.5

0.69

\section{Biases towards female care under strong sexual selection}

Overall, our statistical analysis of the linear mixed-effect model (Model 1) revealed a significant association between sexual selection and the role of sexes in nest building, incubation, and offspring provisioning ( $t=$ $-5.9, p<0.001$; Table 1: Model 1; Figure 2). Despite the consistent pattern of sexual selection being markedly stronger in the 'Female care' than the 'Biparental care' category across all three forms of care, in the stage of nest building, sexual selection scores were the highest in the 'Male care' category $(\mathrm{N}=19$ species). This pattern implies that in the small proportion of Passeriformes where nests were built solely by males, nest building may also function as a means of mate attraction. In addition, the model revealed that 'Female care' was more prevalent in species of larger body size $(t=-3.5, p=0.001$; Table 1: Model 1). Furthermore, sex roles in parental care across three different care forms did not depend on research effort $(t=1.1, p=0.29$; Table 1: Model 1). The random effects 'family' and 'genus' explained $28 \%$ of variation in the response variable, indicating that sex roles in parental care have a phylogenetic signal (Table 1: Model 1). Indeed, the phylogenetic signal was even stronger from the phylogenetically controlled regression model $(\lambda=0.54,95$ CI: $0.39-0.65$, Table S1, Model 1). The estimated direction and degree of the fixed effects (i.e. 'form of care', 'Sexual selection' and 'Research effort') from the phylogenetically controlled regression model were almost the same as the linear mixed-effects model except for the fixed effect 'body size' which turned to be not significant (Table S1: Model 1 vs Table 1: Model 1). 
(a)

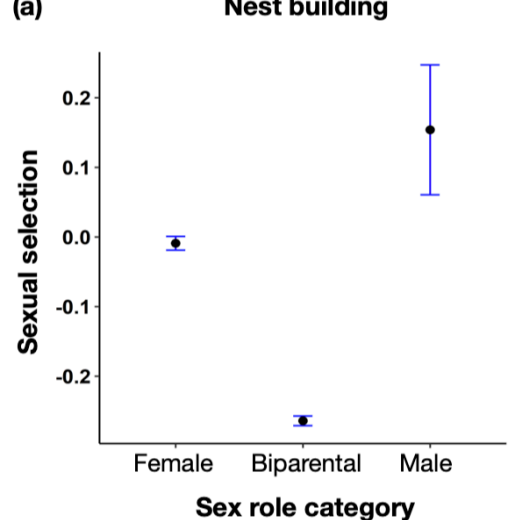

(b)

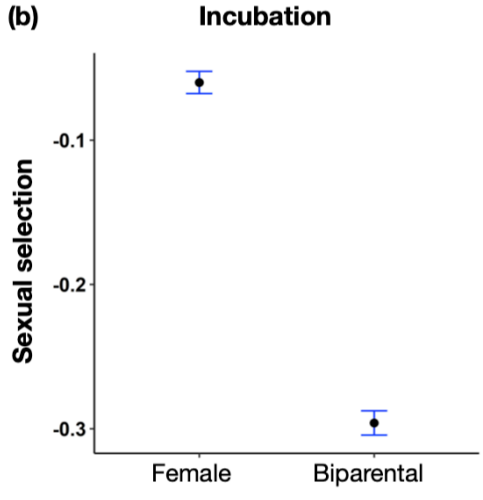

Sex role category

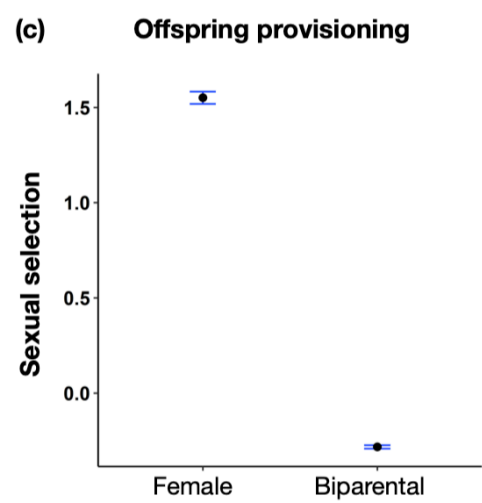

Sex role category

Figure 2. Sexual selection ( $\mathrm{PC1}$ of mating system and sexual dimorphism) scores of three sex role categories in parental care across three distinct care forms. Plots in each panel showed the mean value with $95 \%$ confidence intervals of sexual selection scores in each sex role category.

\section{Association between certainty of paternity and male care}

Model 2 and Model 3 revealed consistently that male in species with high levels of EPP or EPBr tended to show less paternal care (i.e. more 'Female care' and 'Biparental care'; EPP: $t=-2.3, p=0.02$, EPBr: $t=$ $-1.9, p=0.05$; Table 1: Model 2 and Model 3). Those patterns suggested that sexual selection on males and mixed paternity due to female multiple mating disfavour the evolution of male care. In the phylogenetically controlled regression model, the estimated direction and degree of extra-pair paternity (either EPP or EPBr) were stronger and more significant (EPP: $t=-2.6, p=0.01$, EPBr: $t=-2.5, p=$ 0.02; Table S1: Model 2 and Model 3).

\section{No clear association between sex roles in parental care and predation risk}

Model 4 did not show a significant association between sex roles in parental care and nest daily predation rate across three different care forms $(t=-0.2, p=0.9$; Table 1 : Model $4 ; t=0.1, p=1.0$; Table S1: Model 4). Hence, the difference between males and females in the cost of providing care (predation risk in this case) appeared to be non-essential in determining which sex provides care.

\section{No clear association between the number of carers and offspring's life history traits}

Model 5 showed no significant association between the number of carers in each care form and nestlings' developmental time ( $t=0.1, p=0.9$; Table 1: Model 5; $t=0.1, p=0.9$; Table S1: Model 5). There was also no clear association between the number of carers and clutch size $(t=0.5, p=0.6$; Table 1: Model 5; $t=1.6, p=0.1$; Table S1: Model 5). These results suggest that the reproductive value of the current brood (represented by clutch size) and brood needs (represented by both clutch size and nestling developmental time) were not determinant factors of the number of individuals that provide care.

\section{Discussion}


substantially across different care forms (i.e. nest building, incubation, and offspring provisioning). In particular, we identified 21 species where nests were built solely by males, probably as a means of mate attraction. Analyses using a set of linear mixed-effects models identified several ecological and evolutionary factors that may explain sex differences across different forms of parental care. Uniparental care by females tended to be more frequent in species under strong sexual selection, and males were more likely to contribute in species with high certainty of paternity. However, we did not find a significant association between nest predation rate and sex-specific contribution to parental care. There was also no evidence that offspring's life history traits that reflect their reproductive value and brood needs played a role in the number of carers. Our major findings remain unchanged by excluding uncertain species from the dataset (Supplementary Table S2).

\section{Parental care is not a unitary trait regarding which sex provides care}

The distribution of sex role categories differed greatly across the three forms of parental care we studied. During nest building, except a small proportion of species $(2.4 \%, 21$ species) where males contributed alone, biparental care and female-only care occurred at comparable frequencies (52.2\% and 45.5\%). During incubation, female-only care was the most prevalent form (66.3\%) while male-only care was absent. In contrast, during offspring provisioning, biparental care was markedly predominant, with a proportion of $91.8 \%$ and male-only care was also absent. Our results during the offspring provisioning stage were in concordance with a previous survey of Cockburn (2006) [37], where he identified biparental care in $90 \%$ ( $9 \%$ with and $81 \%$ without helpers) of bird species that cover a broad phylogenetic spectrum.

However, we showed that biparental care was no longer the norm in other forms of parental care, and thereby we advocate that parental care should not be treated as a unitary trait, but a composite of several integrated features with diverse functions. For example, in species where males build nests alone and/or defend patches of resources, it is often unclear whether this behaviour should be regarded as a form of parental effort or mating effort [1]. Our finding of particularly strong sexual selection in the 21 species where males build nests alone supports a dual-purpose role of nest building as an investment in both mate attraction and offspring care. The model of Kelly \& Alonzo (2009) [47] showed that males can evolve to allocate proportionally more of their resources to whichever trait (advertisement or parental care) that is more fitness limiting, and if offspring survival is strongly dependent on male care, male advertisement can evolve to be a reliable indicator of parental care. Therefore, it would be interesting to further investigate whether female choice is based on males' different abilities in nest building, and whether offspring survival is strongly dependent on the quality of the nests in the 21 passerine species where nests are built by males only.

We found in this study that sex-specific contribution into parental care can differ greatly across different care forms. Our results and the lack of theoretical predictions highlighted important knowledge gaps in our understanding of parental care as a package with several functionally integrated traits, and how males and females were selected to fulfil different sex roles in the evolutionary time scale. Studies in birds have identified several factors that affect the (relative) contributions of the male and female parents in the 
ecological time scale, including the harshness of abiotic environments, especially temperature and rainfall [48-51], predation risk [52,53], the vulnerability of offspring in the absence of parental care $[54,55]$, and the body condition of the parents themselves [56]. Studies also found that males and female can communicate and negotiate their parental effort [57-61], and the negotiation rules can be sex-specific [62]. Would those factors also play a role in driving sex roles evolution in different care forms in the evolutionary time scale? Do they co-evolve with each other? And how eco-evolutionary feedbacks may affect the evolutionary trajectories and evolutionary transitions? Future work in both empirical and theoretical aspects are needed to answer those questions.

\section{Strong sexual selection was tied to female-biased care}

Our analyses showed a consistent pattern of sexual selection being stronger in species of female-only care than in species of biparental care across three different forms of parental care. This result was in agreement with the Darwin-Bateman paradigm that predicts sexual selection on males leading to the evolution of conventional sex roles [63], and concurred with a recent survey of 659 bird species from 113 families, which found that parental cooperation decreased with the intensity of sexual selection and skewed adult sex ratios [27]. The study of [27] focused on the association between sexual selection and the "inequality" between males and females in parental care contributions, and therefore they recoded the parental care data without sex-specificity (i.e. uniparental care by the male or female were considered equally uncooperative and were both assigned a cooperation score of 0 ; biparental care biased towards either sex were assigned a cooperation score of 1 ; and only when males and females contributed approximately equally, the species were assigned a cooperation score of 2). In addition, although the parental care data of [27] contained eight different parental care activities (corresponding to different care forms in our study), the parental cooperation score was calculated by averaging the statistically centered extent of biparental care across the different activities. In comparison with [27], we covered a narrower phylogenetic spectrum (focusing on passerine birds), but included more species (882 species in total) and associated data on sex-specific contributions of parental care in three distinct forms. Our results were thus complementary to those of [27], and the combined results of these two studies suggest that the role of sexual selection on the evolution of sex-biased parental care may be widespread across avian taxa and across different forms of parental care. Our findings also suggest that sexual selection may play a role in evolutionary transitions between major patterns of parental care, in agreement with previous studies in cichlid fishes [25] and shorebirds [26].

\section{Uncertainty of paternity selected against male care}

Our statistical model showed a significant association between extra-pair paternity and reduced male care across different parental care forms, in agreement with a number of previous comparative studies with a smaller number of species [64-67]. Although theory generally predicts that males should invest more in the care of their genetic offspring and adjust their parental efforts to their share of paternity in the nest [1012,68], empirical support has been mixed, with abundant exceptions where males do not seem to react to the loss of paternity by reducing their parental care efforts. For example, male dunnocks (Prunella modularis) 
did not preferentially feed their genetic offspring in a mixed brood despite that males that had some paternity were more likely to feed the chicks in general [28,29]; male reed buntings (Emberiza schoeniclus) did not adjust their parental effort in relation to EPP, and in mixed paternity nests they did not bias their provisioning to kin [30]; and males western bluebirds (Sialia mexicana) did not reduce parental care even when they observed their mate engaging in extra-pair copulations [31]. Recent theoretical studies revealed some conditions where males may evolve to be insensitive to the loss of paternity, e.g. in cooperative breeding species where offspring help to raise their younger (half-)siblings [69], or in the presence of male alternative reproductive tactics where the "sneaker" males specialize in gaining extra-pair paternity [70]. Empirical studies also found that in species where males were not sensitive to paternity loss, paternal care may not be costly in terms of parental survival [30] and/or the loss of opportunities for siring extra-pair offspring [23]. Few comparative studies (for a rare exception, see [71]) have tested the roles of potential factors that may explain the presence or absence of male response to paternity loss by reducing or withholding paternal care, probably due to a limitation of detailed data on life history traits related to parental care across species. Future efforts in generating and collating such data are therefore indispensable to a better understanding of the relation between certainty of paternity and male investment in parental care.

\section{Nest predation risk did not shape sex roles in parental care}

Our analyses did not show a significant association between nest predation risk and sex differences in parental care. This result was surprising since a survey of 256 species passerine birds showed that the frequency of nest visits decreased as the risk of nest predation increased, because frequent bouts of incubation could increase the visibility of a nest [40], and similar results were found also in seven species of arctic sandpipers [72]. Given that the plumage of females is usually drabber and more cryptic than males, we expected species with high nest predation to show more female-biased care. The lack of correlation could be due to either anti-predatory adaptations, confounding factors that masked the effect of female cryptic plumage, or a combination of both. Species that endure high nest predation risk may have evolved strategies that minimize activities that could attract predators, like long on- and off-bouts of incubation [48], and males with brighter plumage may evolve to attend the nest largely at night when visual predators were inactive, such as in the red-capped plover (Charadrius ruficapillus) [73]. Confounding factors such as nesting site quality and the shape of nests may also override the advantage of drabber plumage of females in providing care. For example, an study using 10 species of open-nesting birds in Arizona, USA revealed a positive correlation between nest predation and parental activity only when nest site effects were considered [74].

\section{Brood needs and offspring's reproductive value did not affect the number of care providers}

Since broods of larger sizes and longer nestling developmental time generally have higher needs, we expected that more carers (i.e. both parents relative to a single parent, or breeders and helpers relative to only the breeders) were required to provide the elevated amount of care. But no such association was found in our data. Our results suggested that the amount of parental care a brood receives may not necessarily increase with the number of carers. Indeed, models have shown that a parent may or may not compensate 
for a reduction of parental effort by the other depending on various factors, including the marginal benefit/harm to offspring as a function of total care received, how well each parent is informed about brood needs, and how well the parents can monitor each other's investment [17,19,20,75]. Negotiation between parents can even produce cases where the offspring do better with one parent than two [18]. Experimental studies by (temporally) removing a parent also showed that the compensation patterns can vary widely from a matching reduction, through no, partial, and full compensation, to even over-compensation [76-79]. Therefore, species are likely to have evolved redundancy in their abilities to provide care, and such abilities could be beneficial to secure reproductive success in cases of losing a partner and/or helper.

\section{Conclusion}

Through a survey of more than 800 species of passerine birds, we found significant variation in terms of which sex provides care in three different forms of parental care. Regarding nest building, except a small proportion of species $(2.4 \%)$ where males build nests alone, the prevalence of female-only care and biparental care were nearly equal. As for incubation, female-only care was twice as frequent as biparental care. Regarding offspring provisioning, biparental care was the predominant pattern, with a prevalence of more than $90 \%$. Our statistical models showed that the intensity of sexual selection may be the primary driving force of the sex-role variation we found in distinct parental care forms. We also found strong support for uncertainly of paternity selecting against male care. As a whole, our results suggest that parental care should not be treated as a unitary trait, but a composite of integrated features with diverse functions. For example, nest building by males may serve a dual function of mate attraction and parental care. Besides those findings, we also identified important knowledge gaps for future theoretical and empirical investigations. For example, we still lack testable theory that make predictions on the relative efforts of male and female parents in different care forms. And we still do not fully understand why males react to a loss of paternity by reducing paternal care in some species but not in others. Would the effects of sexual selection, certainty of paternity, predation risk and offspring life history traits we found in passerine birds be consistent with other avian species? Do other factors, such as adult sex ratio, operational sex ratio, and sex-specific adult mortality, also play a role in shaping sex-role patterns in different forms of parental care? And how do these the driving factors interact with each other in eco-evolutionary feedbacks? Our current work provided a valuable starting point towards answering those new questions. And we encourage future empirical and theoretical studies to go beyond considering parental care as a unitary trait and delve deeper into the components of it, such as different forms and different stages across time.

\section{References}

1. Clutton-Brock TH. 1991 The evolution of parental care. Princeton University Press, Princeton.

2. Royle NJ, Smiseth PT, Kölliker M, editors. 2012 The evolution of parental care. Oxford University Press, Oxford.

3. Székely T, Remeš V, Freckleton RP, Liker A. 2013 Why care? Inferring the evolution of complex 
social behaviour. Journal of Evolutionary Biology 26, 1381-1391.

4. Trivers RL. 1972 Parental investment and sexual selection. In Sexual Selection \& the Descent of Man, pp. 136-179. Aldine Publishing Company, Illinois, USA.

5. Maynard Smith J. 1977 Parental investment: A perspective analysis. Animal Behaviour 25, 1-9.

6. Grafen A, Sibly R. 1978 A model of mate desertion. Animal Behaviour 26, 645-652.

7. Gross MR, Sargent RC. 1985 The evolution of male and female parental care in fishes. American Zoologist 25, 807-822.

8. Andersson M, Wiklund CG, Rundgren H. 1980 Parental defence of offspring: a model and an example. Animal Behaviour 28, 536-542.

9. Winkler DW. 1987 A general model for parental care. The American Naturalist 130, 526-543.

10. Whittingham LA, Taylor PD, Robertson RJ. 1992 Confidence of paternity and male parental care. The American Naturalist 139, 1115-1125.

11. Westneat DF, Sherman PW. 1993 Parentage and the evolution of parental behavior. Behavioral Ecology 4, 66-77.

12. Queller DC. 1997 Why do females care more than males? Proceedings of the Royal Society B 264, $1555-1557$.

13. Lehtonen J, Kokko H. 2012 Positive feedback and alternative stable states in inbreeding, cooperation, sex roles and other evolutionary processes. Philosophical Transactions of the Royal Society B 367,

15. Klug H, Bonsall MB, Alonzo SH. 2013 The origin of parental care in relation to male and female

16. McNamara JM, Wolf M. 2015 Sexual conflict over parental care promotes the evolution of sex

17. McNamara JM, Gasson CE, Houston AI. 1999 Incorporating rules for responding into evolutionary games. Nature 401, 368-371.

18. McNamara JM, Houston AI, Barta Z, Osorno J-L. 2003 Should young ever be better off with one parent than with two? Behavioral Ecology 14, 301-310.

19. Johnstone RA, Hinde CA. 2006 Negotiation over offspring care---how should parents respond to each other's efforts? Behavioral Ecology 17, 818-827. Johnstone RA, Savage JL. 2019 Conditional cooperation and turn-taking in parental care. Frontiers

21. Barta Z, Székely T, Liker A, Harrison F. 2014 Social role specialization promotes cooperation between parents. The American Naturalist 183, 747-761.

22. Henshaw JM, Fromhage L, Jones AG. 2019 Sex roles and the evolution of parental care specialization. Proceedings of the Royal Society B 286, 20191312.

23. Safari I, Goymann W, Kokko H. 2019 Male-only care and cuckoldry in black coucals: does parenting 
hamper sex life? Proceedings of the Royal Society B 286, 20182789.

24. Browning LE, Young CM, Savage JL, Russell DJF, Barclay H, Griffith SC, Russell AF. 2012 Carer provisioning rules in an obligate cooperative breeder: prey type, size and delivery rate. Behavioral Ecology and Sociobiology 66, 1639-1649.

25. Gonzalez-Voyer A, Fitzpatrick JL, Kolm N. 2008 Sexual selection determines parental care patterns in cichlid fishes. Evolution 62, 2015-2026.

26. Olson VA, Webb TJ, Freckleton RP, Szekely T. 2009 Are parental care trade-offs in shorebirds driven by parental investment or sexual selection? Journal of Evolutionary Biology 22, 672-682.

27. Remeš V, Freckleton RP, Tökölyi J, Liker A, Székely T. 2015 The evolution of parental cooperation in birds. Proceedings of the National Academy of Sciences 112, 13603-13608.

28. Burke T, Davies NB, Bruford MW, Hatchwell BJ. 1989 Parental care and mating behaviour of polyandrous dunnocks Prunella modularis related to paternity by DNA fingerprinting. Nature 338, 249-251.

29. Davies NB, Hatchwell BJ, Robson T, Burke T. 1992 Paternity and parental effort in dunnocks Prunella modularis: how good are male chick-feeding rules? Animal Behaviour 43, 729-745.

30. Bouwman KM, Lessells C (Kate) M, Komdeur J. 2005 Male reed buntings do not adjust parental effort in relation to extrapair paternity. Behavioral Ecology 16, 499-506.

31. Dickinson JL. 2003 Male share of provisioning is not influenced by actual or apparent loss of paternity in western bluebirds. Behavioral Ecology 14, 360-366.

32. Dale J, Dey CJ, Delhey K, Kempenaers B, Valcu M. 2015 The effects of life history and sexual selection on male and female plumage colouration. Nature 527, 367-370.

33. Montgomerie RD, Weatherhead PJ. 1988 Risks and rewards of nest defence by parent birds. The Quarterly Review of Biology 63, 167-187.

34. Bowers EK, Jenkins JB, Mueller AJ, Miller KD, Thompson CF, Sakaluk SK. 2019 Conditiondependent begging elicits increased parental investment in a wild bird population. The American Naturalist 193, 725-737.

35. Windt W, Curio E. 1986 Clutch defence in Great Tit (Parus major) pairs and the Concorde fallacy. Ethology 72, 236-242.

36. Billerman SM, Keeney BK, Rodewald PG, Schulenberg TS, editors. 2020 Birds of the World. Cornell Laboratory of Ornithology, Ithaca, NY, USA. (doi:https://birdsoftheworld.org/bow/home)

37. Cockburn A. 2006 Prevalence of different modes of parental care in birds. Proceedings of the Royal Society B 273, 1375-1383.

38. Jetz W, Thomas GH, Joy JB, Hartmann K, Mooers AO. 2012 The global diversity of birds in space and time. Nature 491, 444-448.

39. Brouwer L, Griffith SC. 2019 Extra-pair paternity in birds. Molecular Ecology 28, 4864-4882.

40. Matysioková B, Remeš V. 2018 Evolution of parental activity at the nest is shaped by the risk of nest predation and ambient temperature across bird species. Evolution 72, 2214-2224.

41. Cooney CR et al. 2020 Ecology and allometry predict the evolution of avian developmental durations. 
Nature Communications 11, 2383.

42. Valcu M, Dale J, Griesser M, Nakagawa S, Kempenaers B. 2014 Global gradients of avian longevity support the classic evolutionary theory of ageing. Ecography 37, 930-938.

43. R Core Team. 2021 R: A language and Environment for Statistical Computing. Vienna, Austria: R Foundation for Statistical Computing.

44. Bates D, Mächler M, Bolker B, Walker S. 2015 Fitting linear mixed-effects models using lme4. Journal of Statistical Software 67, 1-48.

45. Ho LST, Ané C. 2014 Intrinsic inference difficulties for trait evolution with Ornstein-Uhlenbeck models. Methods in Ecology and Evolution 5, 1133-1146.

46. Ho LST, Ané C. 2014 A linear-time algorithm for Gaussian and non-Gaussian trait evolution models. Systematic Biology 63, 397-408.

47. Kelly NB, Alonzo SH. 2009 Will male advertisement be a reliable indicator of paternal care, if offspring survival depends on male care? Proceedings of the Royal Society B 276, 3175-3183.

48. Conway CJ, Martin TE. 2000 Evolution of passerine incubation behavior: influence of food, temperature, and nest predation. Evolution 54, 670-685.

49. AlRashidi M, Kosztolányi A, Shobrak M, Küpper C, Székely T. 2011 Parental cooperation in an extreme hot environment: natural behaviour and experimental evidence. Animal Behaviour 82, 235243.

50. Coe BH, Beck ML, Chin SY, Jachowski CMB, Hopkins WA. 2015 Local variation in weather conditions influences incubation behavior and temperature in a passerine bird. Journal of Avian Biology 46, 385-394.

51. Vincze $\mathrm{O}$ et al. 2017 Parental cooperation in a changing climate: fluctuating environments predict shifts in care division. Global Ecology and Biogeography 26, 347-358.

52. Basso A, Richner H. 2015 Effects of nest predation risk on female incubation behavior and offspring growth in great tits. Behavioral Ecology and Sociobiology 69, 977-989.

53. Ghalambor CK, Martin TE. 2002 Comparative manipulation of predation risk in incubating birds reveals variability in the plasticity of responses. Behavioral Ecology 13, 101-108.

54. Dale S, Gustavsen R, Slagsvold T. 1996 Risk taking during parental care: a test of three hypotheses applied to the pied flycatcher. Behavioral Ecology and Sociobiology 39, 31-42.

55. Listøen C, Karlsen RF, Slagsvold T. 2000 Risk taking during parental care: a test of the harm-tooffspring hypothesis. Behavioral Ecology 111, 40-43.

56. Komdeur J, Szentirmai I, Székely T, Bleeker M, Kingma SA. 2005 Body condition and clutch desertion in penduline tit Remiz pendulinus. Behaviour 142, 1465-1478.

57. Sládeček M, Vozabulová E, Brynychová K, Šálek ME. 2019 Parental incubation exchange in a territorial bird species involves sex-specific signalling. Frontiers in Zoology 16, 7.

58. Bulla M, Valcu M, Rutten AL, Kempenaers B. 2019 Temporary mate removal during incubation leads to variable compensation in a biparental shorebird. Frontiers in Ecology and Evolution 7, 93.

59. Dey CJ, O'Connor CM, Balshine S, Quinn JS. 2014 Cooperative males reduce incubation in response 
to cues of female--female competition. Ibis 156, 446-451.

60. Kosztolányi A, Cuthill IC, Székely T. 2009 Negotiation between parents over care: reversible compensation during incubation. Behavioral Ecology 20, 446-452.

61. Hinde CA. 2006 Negotiation over offspring care?---a positive response to partner-provisioning rate in great tits. Behavioral Ecology 17, 6-12.

62. Iserbyt A, Farrell S, Eens M, Müller W. 2015 Sex-specific negotiation rules in a costly conflict over parental care. Animal Behaviour 100, 52-58.

63. Janicke T, Häderer IK, Lajeunesse MJ, Anthes N. 2016 Darwinian sex roles confirmed across the animal kingdom. Science Advances 2, e1500983.

64. Møller AP, Birkhead TR. 1993 Certainty of paternity covaries with paternal care in birds. Behavioral Ecology and Sociobiology 33, 261-268.

65. Møller AP, Cuervo JJ. 2000 The evolution of paternity and paternal care in birds. Behavioral Ecology 11, 472-485.

66. Matysioková B, Remeš V. 2013 Faithful females receive more help: The extent of male parental care

67. Lifjeld JT et al. 2019 Evolution of female promiscuity in Passerides songbirds. BMC Evolutionary Biology 19, 1-14.

68. Alonzo SH. 2010 Social and coevolutionary feedbacks between mating and parental investment. Trends in Ecology and Evolution 25, 99-108.

69. Liedtke J, Fromhage L. 2012 When should cuckolded males care for extra-pair offspring? Proceedings of the Royal Society B 279, 2877-2882.

70. Li X-Y, Morozov A, Goymann W. 2021 Coevolution of female fidelity and male help in populations with alternative reproductive tactics. Proceedings of the Royal Society B 288, 20202371.

71. Griffin AS, Alonzo SH, Cornwallis CK. 2013 Why do cuckolded males provide paternal care? PLoS Biology 11, e1001520.

72. Meyer N et al. 2020 Nest attentiveness drives nest predation in arctic sandpipers. Oikos 129, 14811492.

73. Ekanayake KB, Weston MA, Nimmo DG, Maguire GS, Endler JA, Küpper C. 2015 The bright incubate at night: sexual dichromatism and adaptive incubation division in an open-nesting shorebird. Proceedings of the Royal Society B 282, 2015.

74. Martin TE, Scott J, Menge C. 2000 Nest predation increases with parental activity: separating nest nest attendance compatible with stable biparental care? Behavioral Ecology 13, 838-843.

76. Rauter CM, Moore AJ. 2004 Time constraints and trade-offs among parental care behaviours: effects of brood size, sex and loss of mate. Animal Behaviour 68, 695-702. 
bioRxiv preprint doi: https://doi.org/10.1101/2021.10.20.465177; this version posted November $2,2021$. The copyright holder for this

preprint (which was not certified by peer review) is the author/funder, who has granted bioRxiv a license to display the preprint in perpetuity. It is made available under aCC-BY-NC-ND 4.0 International license.

Differential response by males and females. Animal Behaviour 69, 551-559.

603

78. Suzuki S, Nagano M. 2009 To compensate or not? Caring parents respond differentially to mate removal and mate handicapping in the burying beetle, Nicrophorus quadripunctatus. Ethology 115,

605 $1-6$.

606

79. Harrison F, Barta Z, Cuthill I, Szekely T. 2009 How is sexual conflict over parental care resolved? A 